\title{
Rate constants from instanton theory via a microcanonical approach
}

\author{
Sean R. McConnell, ${ }^{1}$ Andreas Löhle, ${ }^{1}$ and Johannes Kästner ${ }^{1}$ \\ Institute for Theoretical Chemistry, University of Stuttgart, Pfaffenwaldring 55, 70569 Stuttgart,Germany, \\ kaestner@theochem.uni-stuttgart.de
}

(Dated: 9 September 2020)

Microcanonical instanton theory offers the promise of providing rate constants for chemical reactions including quantum tunneling of atoms over the whole temperature range. We discuss different rate expressions, which require the calculation of stability parameters of the instantons. The traditional way of obtaining these stability parameters is shown to be numerically unstable in practical applications. We provide three alternative algorithms to obtain such stability parameters for non-separable systems, i.e., systems in which the vibrational modes perpendicular to the instanton path couple to movement along the path. We show the applicability of our algorithms on two molecular systems: $\mathrm{H}_{2}+\mathrm{OH} \rightarrow \mathrm{H}_{2} \mathrm{O}+\mathrm{H}$ using a fitted potential energy surface and $\mathrm{HNCO}+\mathrm{H} \rightarrow \mathrm{NH}_{2} \mathrm{CO}$ using a potential obtained on-the-fly from density functional calculations.

\section{INTRODUCTION}

The calculation of reaction rates is a longstanding challenge in computational chemistry ${ }^{115}$ At low temperatures, quantum tunneling of atoms must be taken into account ${ }^{6]}$ Instanton theory is emerging as a promising and frequently used method to calculate tunneling rates in moderately-sized chemical reactions. It originated in the 1960s and 1970s in somewhat different formulations ${ }^{47-17}$ It is mostly used with a canonical ensemble, which assumes thermal equilibration at each stage of the reaction and provides thermal rate constants. However, even in its early stages a microcanonical formulation, with rate constants depending on the energy, was provided. 9 This turned out to be rarely used. More recently, the connection between instanton theory and Ring-polymer molecular dynamics was shown ${ }^{[18] 19}$ and the rate expressions were derived from first principles. 20 While the location of an instanton path, an unstable periodic orbit, used to be a daunting and numerically unstable procedure, recently improved algorithms to search for instantons were proposed. The problem of finding an instanton path can be turned into a saddle-point search problem $^{21} 23$ for which quantum chemistry has a rich variety of methods at hand. It turned out that a simple modification of a truncated Newton search converges very fast and is stable even for somewhat noisy gradients and Hessians of the potential energy ${ }^{2425}$ This allowed the location of instantons in systems with up to 78 active atoms. ${ }^{[26}$ Meanwhile, the canonical version of semiclassical instanton theory is frequently used to calculate thermal rate constants. $22|24| 26 \sqrt[49]{2}$

However, for bimolecular reactions it is often desirable to assume a canonical ensemble only for the separated reactant states but not during any stage of the reaction. Specifically, many bimolecular reactions exhibit a pre-reactive energy minimum, a weakly bound Van-derWaals complex. At low pressure, such a complex does not thermally equilibrate and either proceeds over the transition state or decays again. This limits the applicability of canonical instanton theory $\sqrt[34 \mid 45]{ }$ A microcanonical formulation allows the use of the reactant's thermal distribution to calculate thermal rate constants without assuming thermalization in a pre-reactive minimum.

Moreover, canonical instanton theory is only applicable up to a crossover temperature $T_{\mathrm{c}}$, the temperature where the instanton path collapses to a point. While different approaches to extend the formulation above $T_{\mathrm{c}}$ have been suggested, 50/53 a microcanonical formulation provides thermal rate constants at all temperatures naturally. $\underline{54}$

Even though algorithms to calculate microcanonical instanton rate constants were proposed decades agd 0 they were rarely used for real chemical reactions in which the vibrational modes are not separable from each other and, most importantly, from the transition mode. The reason for this is that these approaches lacked numerical stability. In this paper we propose different algorithms to calculate microcanonical instanton rate constants for non-separable systems. We compare results to canonical instanton theory, as well as to results from exact quantum dynamics.

The paper is organized as follows: First, we briefly review the theory of microcanonical rate constants and ways to derive the cumulative reaction probability $P(E)$ using instanton theory and the semiclassical approximation. The resulting rate expressions require the calculation of stability parameters. Besides the traditional approach of solving the stability matrix differential equation, we provide three alternative, numerically more stable, approaches to calculate the stability parameters. In the applications section we apply these to the two test cases $\mathrm{H}_{2}+\mathrm{OH} \rightarrow \mathrm{H}_{2} \mathrm{O}+\mathrm{H}$ and $\mathrm{HNCO}+\mathrm{H} \rightarrow \mathrm{NH}_{2} \mathrm{CO}$. Finally we discuss advantages and disadvantages of our newly proposed approaches and of microcanonical instanton theory in general.

\section{THEORY}

\section{A. Microcanonical Reaction Rate Constants}

In order to describe bimolecular reactions to their full extent one would have to solve the Schrödinger equa- 
tion with proper scattering boundary conditions in order to obtain the S-matrix which contains all the necessary information to calculate the state-to-state differential and integral cross sections. By averaging over all cross sections one obtains the so called cumulative reac-

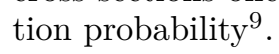

$$
\left.P(E)=\sum_{J}(2 J+1) \sum_{n_{p}, n_{r}} \mid \mathbf{S}_{n_{p}, n_{r}}(E, J)\right)\left.\right|^{2}
$$

whereby $n_{p}$ and $n_{p}$ denote the quantum numbers of the product and reactant state, $J$ the total angular momentum and $E$ the total energy of the system. The microcanonical rate constant $k(E)$ can then be calculated as follows $\sqrt{55}$

$$
k(E)=\frac{1}{2 \pi \hbar} \frac{P(E)}{\Gamma_{r}(E)}
$$

where $\Gamma_{r}(E)$ is density of states in the reactant state. The canonical rate constant $k(T)$ is obtained via a Laplace transform of $P(E)$

$$
k(T)=\frac{1}{2 \pi \hbar Q_{\mathrm{RS}}} \int_{-\infty}^{\infty} P(E) \exp (-\beta E) d E
$$

divided by the canonical partition function $Q_{\mathrm{RS}}$ of the reactant state per unit volume. Here $\beta$ is the inverse temperature, $\beta=1 /\left(k_{\mathrm{B}} T\right)$.

Since in most cases one is only interested in obtaining the chemical reaction rate rather than detailed information about all state to state interactions (which would be provided by a full scattering calculation) an efficient way to obtain $P(E)$ directly is the use of the quantum flux-flux autocorrelation formalism ${ }^{56}$ which gives an exact expression for $P(E)$

$$
P(E)=2 \pi \hbar \operatorname{tr}\left(\delta(E-\hat{H}) \hat{F} \hat{P}_{r}\right)
$$

where $\delta(E-\hat{H})$ is the density operator in the microcanonical ensemble. $\hat{F}$ is the quantum mechanical analogue of the classical flux function which counts the number of elementary reactions from reactant to product and is given by

$$
\hat{F}=\frac{\mathrm{i}}{\hbar}[\hat{H}, \hat{\theta}(s)]
$$

where $\hat{\theta}$ is the Heaviside step function and $s$ denotes a function that is negative on the reactant side of the dividing surface and positive on the product side. The projection operator $\hat{P}_{r}$ is given by the time evolved Heaviside function in the limit of $t \rightarrow \infty$

$$
\begin{aligned}
\hat{P}_{r} & =\lim _{t \rightarrow \infty} e^{\frac{i}{\hbar} \hat{H} t} \hat{\theta}(s) e^{-\frac{i}{\hbar} \hat{H} t} \\
& =\int_{0}^{\infty} e^{\frac{i}{\hbar} \hat{H} t} \hat{F} e^{-\frac{i}{\hbar} \hat{H} t} d t
\end{aligned}
$$

and can be written as the time integral of the time evolved flux operator. It describes the probability that the trajectory remains on the the product side as $t$ approaches infinity. After some manipulations of equation (4) one arrives at the final expression for the cumulative reaction probability 57

$$
P(E)=2 \pi^{2} \hbar^{2} \operatorname{tr}(\delta(E-\hat{H}) \hat{F} \delta(E-\hat{H}) \hat{F})
$$

Over the years there have been several methods 55156158159 proposed to evaluate equation (8) which vary significantly in terms of accuracy and computational effort. However, for large systems, a semi-classical approximation of equation (8) remains the method of choice. In this paper we use a formulation of instanton theory to evaluate $P(E)$ which has been recently proposed by Richardson based on the previous works of Miller in which the evaluation of equation (8) is reduced to finding closed orbits in imaginary time and the calculation of its stability parameters $u_{i} \cdot 60$

\section{B. The Cumulative Reaction Probability $P(E)$}

We consider scattering problems, i.e. situations in which the reactant state is unbound and can adopt a continuum of energy values. This corresponds to a bimolecular reaction. The thermal rate constant $k(\beta)$ can be obtained from the cumulative reaction probability $P(E)$ via

$$
k(\beta) Q_{\mathrm{RS}}(\beta)=\frac{1}{2 \pi \hbar} \int_{E_{\mathrm{RS}}}^{\infty} P(E) \exp (-\beta E) d E
$$

where $E_{\mathrm{RS}}$ is the energy of the reactant state in its vibrational ground state, $Q_{\mathrm{RS}}$ is the partition function of the reactant state per unit volume and $\beta$ is the inverse temperature, $\beta=1 /\left(k_{\mathrm{B}} T\right)$. Atomic units with $\hbar=m_{\mathrm{e}}=4 \pi \epsilon_{0}=1, c=1 / \alpha$ will be used from now on.

In one dimension, $P(E)$ can be obtained by a variety of methods, including a direct numerical solution of Schrödinger's equation. Instanton theory provides an expression for $P(E)$ for a system with $D$ vibrational degrees of freedom: [

$$
\begin{aligned}
P(E)= & \sum_{k=1}^{\infty}(-1)^{k-1} \exp \left(-k S_{0}(E)\right) \times \\
& \prod_{i=1}^{D-1} \frac{1}{2 \sinh \left(k u_{i}(E) / 2\right)}
\end{aligned}
$$

Where $S_{0}$ is the shortened action and $u_{i}(E)$ are the stability parameters of the instanton path. Their dependence on $E$ for a specific model system is displayed in Fig. 1. $D$ is the number of vibrational degrees of freedom of the system. The shortened action is

$$
S_{0}\left(E_{b}\right)=\int_{0}^{T_{0}}\left|\frac{d \mathbf{y}(\tau)}{d \tau}\right|^{2} d \tau=\sqrt{8} \int_{r_{a}}^{r_{b}} \sqrt{E(r)-E_{b}} d r
$$




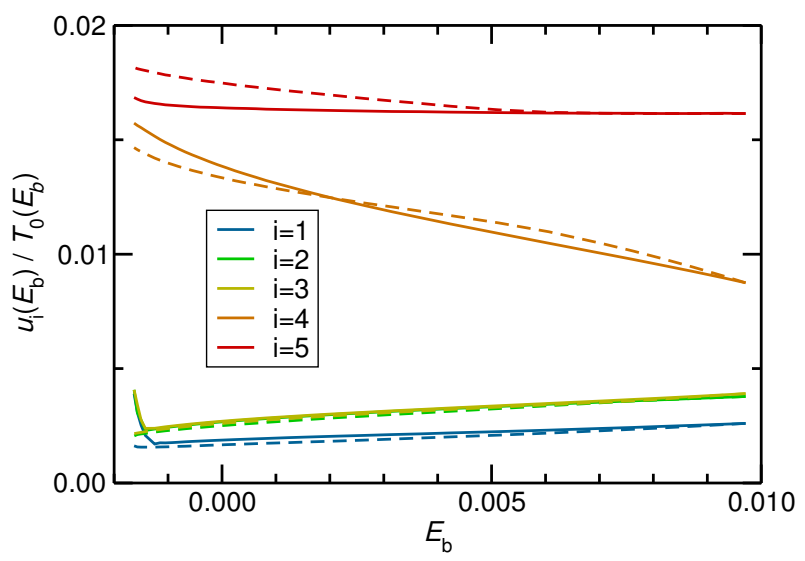

FIG. 1. The stability parameters $u_{i}(E)$ for the reaction $\mathrm{H}_{2}+\mathrm{OH} \rightarrow \mathrm{H}_{2} \mathrm{O}+\mathrm{H} \rightarrow \mathrm{H}_{2} \mathrm{O}+\mathrm{H}$ discussed in the results section. All values in atomic units. Solid lines were obtained by solving the stability matrix differential equation, dashed lines by eigenvalue tracing.

with the integration being done along the instanton path $\mathbf{y}(\tau)$ in mass-weighted coordinates $\mathbf{y}$. The integral on the left in equation (11) is performed in complex time $t \rightarrow i \tau$. In real-space the integration can be done via the arc length $r$ between the turning points $r_{a}$ and $r_{b}$ with $E\left(r_{a}\right)=E\left(r_{b}\right)=E_{b}$. The instanton optimization provides a tunneling energy $E_{b}$ for a given $T_{0}$.

With the hyperbolic sine expressed as its series expansion, equation (10) results in:

$$
\frac{1}{2 \sinh \left(k u_{i}(E) / 2\right)}=\sum_{n_{i}=0}^{\infty} \exp \left[-k\left(n_{i}+\frac{1}{2}\right) u_{i}(E)\right]
$$

from which we arrive at

$$
P(E)=\sum_{n} \sum_{k=1}^{\infty}(-1)^{k-1} \mathrm{e}^{-k\left[S_{0}+\sum_{i=1}^{D-1}\left(n_{i}+\frac{1}{2}\right) u_{i}(E)\right]}
$$

where the short-hand notation

$$
\sum_{n}=\sum_{n_{1}=0}^{\infty} \sum_{n_{2}=0}^{\infty} \ldots \sum_{n_{D-1}=0}^{\infty}
$$

is used. The sum over $k$ can be interpreted as a geometric series $^{9}$ and can be calculated explicitly:

$$
P(E)=\sum_{n}\left\{1+\exp \left[S_{0}(E)+\sum_{i=1}^{D-1}\left(n_{i}+\frac{1}{2}\right) u_{i}(E)\right]\right\}^{-1}
$$

Applicability of the equation is limited in multidimensional systems, since we need $P(E)$ for energies above the energy of the reactant including the zero-point vibrational energy (ZPE) $E_{\mathrm{RS}, \mathrm{ZPE}} \cdot S_{0}(E)$, however, is only available for $E<E_{\mathrm{TS}}$, i.e., the energy of the saddle point without ZPE. In general, for multidimensional systems,
$E_{\mathrm{TS}}$ is often smaller than $E_{\mathrm{RS}, \mathrm{ZPE}}$. However, at least for systems where the vibrational modes are separable for the whole instanton path, the physical background makes it clear that $P(E)$ should be independent of the vibrational frequencies perpendicular to the transition mode, and, thus, the ZPE $\underline{54}$

A way to circumvent that dilemma which is also applicable to non-separable systems was proposed a long time ago, ${ }^{9 \mid 27}$ resulting in

$$
E_{n}=\sum_{i=1}^{D-1}\left(n_{i}+\frac{1}{2}\right) \frac{u_{i}\left(E-E_{n}\right)}{T_{0}\left(E-E_{n}\right)}
$$

and

$$
P(E)=\sum_{n} \frac{1}{1+\exp \left[S_{0}\left(E-E_{n}\right)\right]} .
$$

With that transformation, $S_{0}$ and $u_{i}$ are required in the energy range where they can be easily calculated. Equation (16) needs to be solved iteratively. The individual terms of equation (17) and the sum are displayed in Fig. 2.

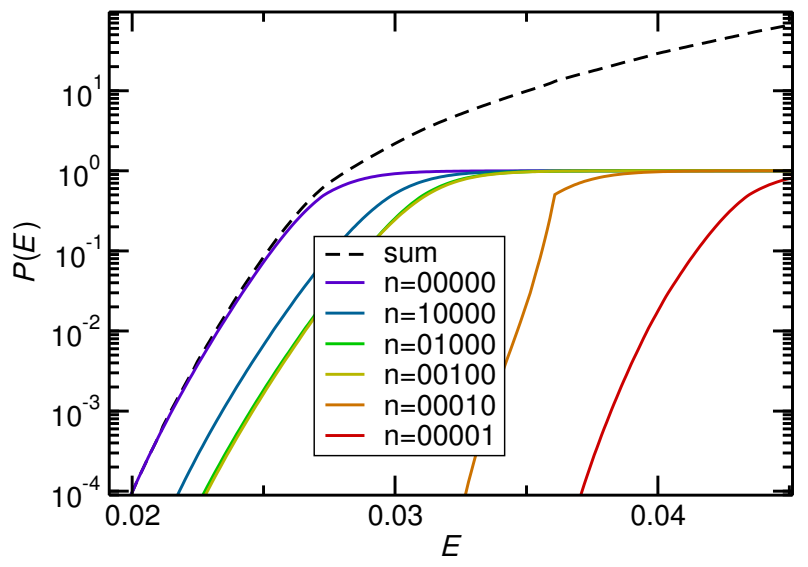

FIG. 2. The individual terms of equation (17) and their sum, $P(E)$, with the stability parameters calculated by eigenvalue tracing. All values in atomic units. It is notable that the energy-dependence of the contribution with $u_{4}$ differs from the others. This is the stability parameter which depends strongly on $E$, see Fig. 1 .

At the energy $E_{\mathrm{TS}}=E_{\mathrm{c}}-E_{n}$ the instanton collapses to one point, $S_{0}=0$ and $u_{i}=T_{0, \mathrm{c}} \omega_{i, \mathrm{TS}}$ with $T_{0, \mathrm{c}}$ being the inverse critical temperature $T_{0, \mathrm{c}}=\beta_{c}=2 \pi / \bar{\omega}_{\mathrm{TS}}$ and $\bar{\omega}_{\mathrm{TS}}$ is the absolute value of the imaginary frequency at the transition state. Above that energy, $P(E)$ is not accessible any more from instanton theory. Instead, we follow a previous suggestion ${ }^{54}$ and use the exact transmission coefficient of a parabolic barrier with the barrier frequency $\bar{\omega}_{\mathrm{TS}} ! \underline{61}$

$$
P_{\text {parabolic }, n}(E)=\frac{1}{1+\exp \left[2 \pi\left(E_{\mathrm{TS}}+E_{n}-E\right) / \bar{\omega}_{\mathrm{TS}}\right]}
$$


Almost indistinguishable results are obtained when using the transmission coefficient of a symmetric Eckart barrier with the same barrier height and frequency as the real barrier. This approach may lead to a kink in the contribution to $P(E)$, as visible, for example, for $n=(00010)$ in Fig. 2 (orange line), which will be averaged out, however, when calculating $k(T)$.

As discussed below, for non-separable systems at low temperature, the calculation of the individual stability parameters $u_{i}$ can be numerically unstable. In these cases it is often still possible to calculate the term as a multidimensional integral $51|62| 63$

$$
\begin{gathered}
\exp (-\sigma)=\prod_{i=1}^{D-1} \frac{1}{2 \sinh \left(u_{i}(E) / 2\right)}=\prod_{i=1}^{D-1} \int d \mathbf{Y}_{\perp}{ }^{(i)}(\tau) \times \\
\exp \left[-\int_{0}^{T_{0}} \mathbf{Y}_{\perp}{ }^{(i)}(\tau)^{T}\left(-\frac{1}{2} \frac{d^{2}}{d \tau^{2}}+\frac{1}{2} \mathbf{V}^{\prime \prime}(\tau)\right) \mathbf{Y}_{\perp}{ }^{(i)}(\tau) d \tau\right]
\end{gathered}
$$

Here, the matrix $\mathbf{Y}_{\perp}(\tau)$ is a co-moving basis containing all vibrational modes orthogonal to the instanton path, $\mathbf{Y}_{\perp}{ }^{(i)}(\tau)$ is the $i$-th column vector of $\mathbf{Y}_{\perp}(\tau)$ and $\mathbf{V}^{\prime \prime}$ is the matrix of second derivatives of the potential energy with respect to mass-weighted Cartesian coordinates. $P(E)$ can then be approximated by

$$
P(E)=\sum_{n} \frac{1}{1+\exp \left[S_{0}\left(E-E_{\mathrm{vib}, n}-\sigma / T_{0}\right)\right]}
$$

with

$$
E_{\mathrm{vib}, n}=\sum_{i=1}^{D-1} \omega_{i, \mathrm{TS}} n_{i}
$$

where $\sigma / T_{0}$ approximates the zero-point vibrational energy and $E_{\mathrm{vib}, n}$ covers vibrational excitations. In many cases, the first term of equation (20), the one with $E_{\mathrm{vib}, n}=0$, dominates. A similar approach has been suggested recently! $\underline{54}$

In a numerical implementation, instantons are optimized for a given set of oscillation times $T_{0}$ (or temperatures $\left.\beta=T_{0} / \hbar\right)$. These provide sets of $S_{0}, E_{b}, u_{i}(E)$ or $\sigma(E)$. The properties required to calculate $P(E)$ for a given energy $E$ are interpolated. When individual stability parameters are used, $u_{i}(E) / T_{0}(E)$ is linearly interpolated to iteratively solve equation $(16)$. Then $S_{0}$ is linearly interpolated to obtain $P(E)$ via equation (17). If equation (20) is used, then $S_{0}$ is linearly interpolated between the two neighboring occurrences of $E=E_{b}+\sigma / T_{0}$.

At low energy and for large vibrational frequencies, the sum over $n$ in equation (17) can be truncated after a few terms, possibly even after the first term. At high energies, especially when $E>E_{\mathrm{TS}, \mathrm{ZPE}}$, many terms must be included. At high enough energies, however, $S_{0}=0$ and can be assumed independent of $E_{n}$. The quantization of vibrational energy levels can therefore be neglected, which results in

$$
P(E)=\frac{\left(E-E_{\mathrm{TS}}\right)^{D-1}}{(D-1) !} \prod_{i=1}^{D-1}\left(\omega_{i, \mathrm{TS}}\right)^{-1} \quad \text { for } E \gg E_{\mathrm{TS}}
$$

We use equation 22 for energies above $E_{\mathrm{TS}, \mathrm{ZPE}}+10 \times$ $\omega_{\mathrm{TS}, \mathrm{min}}$, with $\omega_{\mathrm{TS}, \mathrm{min}}$ being the smallest vibrational frequency at the transition state perpendicular to the transition mode. This ensures that the fist 10 quanta of the vibrations are taken into account explicitly and the continuous expression is used above.

Up to now, we have only discussed the treatment of vibrational levels. We consider the rotational motion to be separable from the internal motion. Our $P(E)$ is essentially $P(E, J)$ for $J=0$. In the $J$-shifting approximation $\frac{6465}{65}$ the rotation-dependence of $P(E, J)$ is taken out of the integral in equation $(9)$ :

$$
\begin{aligned}
k(\beta) Q_{\mathrm{RS}}(\beta)= & \frac{1}{2 \pi} Q_{\mathrm{rot}, \mathrm{TS}} \times \\
& \int_{E_{\mathrm{RS}}}^{\infty} P(E, J=0) \exp (-\beta E) d E
\end{aligned}
$$

The rotational partition function of the transition state $Q_{\text {rot,TS }}$ is approximated by is classical expression. This is generally a good approximation. The moments of inertia are obtained from the transition state geometry.

In practice, instantons are located at pre-defined $T_{0}$ (or temperatures) by sequential cooling. To cover the full temperature range, instantons need to be located until $E_{b}+\sigma / T_{0}<E_{\mathrm{ZPE}, \mathrm{RS}}$. The thermal rate is then obtained via

$$
k(\beta)=\frac{1}{2 \pi} \frac{Q_{\mathrm{rot}, \mathrm{TS}}}{Q_{\mathrm{RS}}(\beta)} \int_{E_{\mathrm{ZPE}, \mathrm{RS}}}^{\infty} P(E) \exp (-\beta E) d E
$$

\section{The Stability Parameters $u_{i}$}

Using equations (16) and (17) or equation 20 require the calculation of the stability parameters $u_{i}$ or at least of their combination in the form of $\sigma$. Methods to calculate these have appeared in the literature. They almost exclusively consist of integrating the stability matrix differential equation. ${ }^{966} 68$ However, it is clear that this approach is numerically unstable for strong coupling between the modes and/or for a small number of images $P$ discretizing the instanton path. The number of images must be kept small, though, to keep the computational effort at bay when dealing with energies and its derivatives calculated on the fly. We derived and tested several approaches to calculate $u_{i}(E)$ or $\sigma(E)$ and here report on the four that proved numerically most stable in practice.

All the algorithms described here were implemented in a development version of the open-source general-purpose geometry optimizer DL-FIND! ${ }^{69}$ The code will be made available to the scientific community in due course. 


\section{The stability matrix differential equation}

The stability parameters $u_{i}$ are found by solving the linearized equations of motion for the stability matrix $\mathbf{R}(\tau) \underline{960 / 70}$

$$
\frac{d}{d \tau} \mathbf{R}(\tau)+\mathbf{F}(\tau) \mathbf{R}(\tau)=0
$$

where $\mathbf{R}$ is a $2 D \times 2 D$ matrix and

$$
\mathbf{F}(\tau)=\left(\begin{array}{cc}
\mathbf{0} & -\mathbf{1} \\
-\mathbf{V}^{\prime \prime}(\tau) & \mathbf{0}
\end{array}\right)
$$

The matrix $\mathbf{V}^{\prime \prime}$ is the matrix of second derivatives of the potential energies with respect to the mass-weighted coordinates of the atoms at the point $\tau$ along the instanton path. Equation 25 must be solved for $\mathbf{R}\left(T_{0}\right)$ with the initial condition $\mathbf{R}(0)=\mathbf{1}$. The eigenvalues of $\mathbf{R}\left(T_{0}\right)$ are in pairs $e^{u_{i}}$ and $e^{-u_{i}}$ for each $i$. Besides the $D-1$ stability parameters, $\mathbf{R}\left(T_{0}\right)$ has two additional eigenvalues which are unity and correspond to the movement along the path. For a molecular system, there are additional 10 (for linear molecules) or 12 eigenvalues of $\mathbf{R}\left(T_{0}\right)$ which are unity and correspond to the translation and rotation of the total system. In practice, equation 25 is solved with an implicit (or backward) Euler algorithm or, alternatively, a fourth-order Runge-Kutta approach (RK4), by discretization using the images of the instanton path. RK4 is used in the results section unless noted otherwise. Solving the stability matrix is a reliable technique when instanton paths are short. At lower energies, depending on the number of images $P$, the eigenvalues which correspond to movement along the path become indistinguishable from the $u_{i}$ and the algorithm becomes numerically unstable, as can be seen in Fig. 1 by the increase of $u_{i}(E) / T_{0}(E)$ for the lowest three stability parameters (blue, green and yellow solid curves) at low energies. Applicability of the method can be extended by using solely $e^{u_{i}}$ and ignoring $e^{-u_{i}}$, which becomes small and may become negative due to numerical noise. At too low energies, eigenvalues which are supposed to be used to calculate $u_{i}$ show non-zero imaginary parts. In these cases, we extrapolate by using $u_{i}(E)$ from the lowest energy for which valid $u_{i}$ were obtained.

\section{Stability parameters by eigenvalue tracing}

An approximation to equations (25) and (26) can be found by realizing that for slowly-varying frequencies, the stability parameters $u_{i}(E)$ can be interpreted as frequencies $\omega_{i}(\tau)$ perpendicular to the instanton path averaged along that path,

$$
u_{i}(E)=\int_{0}^{T_{0}} \omega_{i}(\tau) d \tau
$$

To achieve that averaging, individual vibrational frequencies need to be traced along the instanton path and then averaged. To do this, we first construct a reduced $(D-1) \times(D-1)$ Hessian matrix $\tilde{\mathbf{V}}^{\prime \prime}$ at each image of the instanton. This is found by

$$
\tilde{\mathbf{V}}^{\prime \prime}=\mathbf{Y}_{\perp}^{T} \mathbf{V}^{\prime \prime} \mathbf{Y}_{\perp}
$$

i.e., projecting the full Hessian onto a basis $\mathbf{Y}_{\perp}$ which contains all modes perpendicular to the instanton path at that image and perpendicular to the translational and rotational eigenvectors. The tangent vector of the instanton path is provided by the eigenvector $v_{\text {tang }}$ of the Hessian of the full instanton, the eigenvalue of which is zero. The basis $\mathbf{Y}_{\perp}$ does not contain the mode corresponding to $v_{\text {tang }}$, hence its shape $D \times(D-1)$. This eigenvector $v_{\text {tang }}$ provides the tangent of each image of the instanton. The translational and rotational eigenvectors are constructed as described elsewhere. ${ }^{71}$

In order to average the eigenvectors to obtain $u_{i}(E)$, equivalent modes need to be traced along the instanton path. Such a tracing is possible if $\tilde{\mathbf{V}}^{\prime \prime}$ is constructed on a carefully chosen coordinate system. A Gram-Schmidt process is used to generate $\mathbf{Y}_{\perp}$ at an arbitrary starting coordinate and to orthogonalize all $D-1$ components of $\mathbf{Y}_{\perp}$ to the tangential vector and all unit vectors of rotation and translation. An initial guess basis is supplied to the Gram-Schmidt algorithm which can be arbitrarily chosen for the first image. The eigenvectors of $\tilde{\mathbf{V}}^{\prime \prime}$ are found and saved in this reduced basis. For the neighboring image, the process is repeated, new vectors tangential to the path and for rotation and translation are found and a guess must be provided for the remaining $D-1$ vectors, this time the set of $D-1$ saved eigenvectors of the previous step having the smallest projection on to the instanton path are used as the new guess vectors. This ensures that the Gram-Schmidt process produces a new coordinate system which is similar to the coordinate system of the previous step. The eigenvectors of neighboring $\tilde{\mathbf{V}}^{\prime \prime}$ are represented in roughly similar orthogonal bases, making their eigenvector comparison possible. The maximum of the dot-products between eigenvectors of successive images indicate the connection of the modes along the instanton paths. This process is repeated for all images along the path. The stability parameter $u_{i}(E)$ is then simply the arithmetic average of the square roots of the eigenvalue of mode $i$. Application of the eigenvalue tracing along the instanton path is shown in Fig. 3

Equation (27) is only exact for separable systems and for a collapsed instanton. In non-separable systems it approximates equations (25) and (26) quite well as it can be seen in Fig. 1] An obvious problem with eigenvalue tracing arises when too few images are used to localize the instanton path. If there is a loss of coherence between neighbouring $\tilde{\mathbf{V}}^{\prime \prime}$ then modes which should be identified as connected/distinct may be misclassified. The consequence of this can be seen in Fig. 3, the where the crossings might switch to an avoided crossing. 


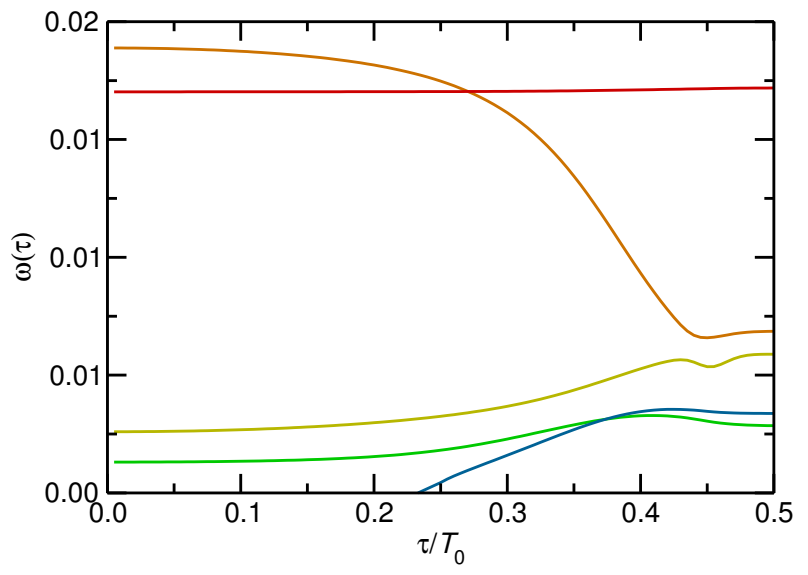

FIG. 3. Vibrational frequencies of perpendicular modes traced along the instanton path for the reaction $\mathrm{H}_{2}+\mathrm{OH}$ $\rightarrow \mathrm{H}_{2} \mathrm{O}+\mathrm{H}$ on the $\mathrm{NN1}{ }^{172}$ potential energy surface.

\section{Frequency averaging to approximate $\sigma$}

Especially for a low number of images $P$, eigenvalue tracing becomes numerically unstable. In order to approximate $\sigma$, however, it is sufficient to know the trace of the square root of the Hessian matrix, which will be justified in the following. For large frequencies or large $T_{0}, 2 \sinh \left(u_{i} / 2\right)$ can be approximated by $\exp \left(u_{i} / 2\right)$ which turns equation 19 into

$$
\sigma(E) \approx \sum_{i=1}^{D-1} \frac{u_{i}(E)}{2}
$$

To obtain this, we consider the second line of equation (19), and recognize the term in round brackets, $-\frac{1}{2} \frac{d^{2}}{d \tau^{2}}+\frac{1}{2} \mathbf{V}^{\prime \prime}(\tau)$, is the Hamiltonian for quantum harmonic oscillators. This can be replaced with a diagonal energy-eigenvalue matrix $\boldsymbol{\Omega}(\tau)$

$$
\mathbf{Y}_{\perp}{ }^{(i)}(\tau)^{T}\left(-\frac{1}{2} \frac{d^{2}}{d \tau^{2}}+\frac{1}{2} \mathbf{V}^{\prime \prime}(\tau)\right) \mathbf{Y}_{\perp}{ }^{(i)}(\tau) \rightarrow \frac{\boldsymbol{\Omega}(\tau)_{i i}}{2}
$$

The matrix $\boldsymbol{\Omega}(\tau)$ does not contain the eigenvalues of $\mathbf{V}^{\prime \prime}(\tau)$, but rather the eigenvalues of the reduced Hes$\operatorname{sian} \tilde{\mathbf{V}}^{\prime \prime}(\tau)$. In the limit $T_{0} \rightarrow \infty$, only the ground states of the quantum harmonic oscillators make a significant contribution to the integral in equation (19), thus the elements of $\boldsymbol{\Omega}(\tau)$ are $\boldsymbol{\Omega}(\tau)_{i j}=\delta_{i j} \tilde{\omega}_{i}(\tau)$. Note, that $\tilde{\omega}_{i}$ are the energy-eigenvalues, i.e., the square roots of the eigenvalues of $\tilde{\mathbf{V}}^{\prime \prime}(\tau)$. Using the replacement in equation 30 , we discretize the integral over $d \tau$ in equation 119 which may be rewritten

$$
\begin{aligned}
e^{\sigma} & =\prod_{i=1}^{D-1} \int d \mathbf{Y}_{\perp, \mathbf{0}}{ }^{(i)} \mathbf{Y}_{\perp, \mathbf{0}}{ }^{(i) T}\left[e^{\sum_{j=1}^{P}\left(\frac{\boldsymbol{\Omega}_{i i}^{(j)}}{2}\right) \Delta \tau}\right] \mathbf{Y}_{\perp, \mathbf{0}}{ }^{(i)}, \\
e^{\sigma} & =\prod_{i=1}^{D-1} \prod_{j=1}^{P} \int d \mathbf{Y}_{\perp, \mathbf{0}}{ }^{(i)} \mathbf{Y}_{\perp, \mathbf{0}}{ }^{(i) T}\left[e^{\Delta \tau \frac{\boldsymbol{\Omega}_{i i}^{(j)}}{2}}\right] \mathbf{Y}_{\perp, \mathbf{0}}{ }^{(i)} .
\end{aligned}
$$

where $\mathbf{Y}_{\perp, \mathbf{0}}$ is an arbitrary, fixed, perpendicular basis. We are left with the definition of an operator trace. This formulation for $\sigma$ has been used elsewhere.

$$
\begin{aligned}
e^{\sigma} & =\int d \mathbf{Y}_{\perp, \mathbf{0}}\left\langle\mathbf{Y}_{\perp, \mathbf{0}}\left|e^{\sum_{j=1}^{P} \Delta \tau \frac{\boldsymbol{\Omega}^{(j)}}{2}}\right| \mathbf{Y}_{\perp, \mathbf{0}}\right\rangle, \\
\sigma & \approx \frac{T_{0}}{2 P} \sum_{j=1}^{P} \operatorname{tr}\left(\boldsymbol{\Omega}^{(j)}\right)
\end{aligned}
$$

We see that equation 32 corresponds exactly to equation (29) given the trace is conducted over $D-1$ dimensions and if the $P$ images are spaced evenly in imaginary time.

In practice, the eigenvalues $\tilde{\omega}_{i}$ of $\tilde{\mathbf{V}}^{\prime \prime}$ don't need to be calculated explicitly. The square roots $\omega_{n, j}$ of the eigenvalues of the Hessian-matrices $\mathbf{V}^{\prime \prime}{ }_{j}$ of all images $j$ are calculated. For each image, all $D$ of these $\omega_{n}(\tau)$ which belong to any vibrations are summed up. In order to remove the contribution along the path, $\sigma$ is then obtained as

$$
\sigma \approx \frac{T_{0}}{2 P} \Re\left[\sum_{j=1}^{P} \sum_{n=1}^{D} \omega_{n, j}-\sqrt{\left\langle v_{\text {tang }, j}\left|\mathbf{V}^{\prime \prime}{ }_{j}\right| v_{\text {tang }, j}\right\rangle}\right]
$$

with $v_{\text {tang, } j}$, again, being the tangent of the instanton path at image $j$. It may be that $\omega_{n, j}$ is imaginary or $\left\langle v_{\text {tang, } j}\left|\mathbf{V}^{\prime \prime}{ }_{j}\right| v_{\text {tang, }, j}\right\rangle$ is negative. In both cases, these contributions need to be ignored, only the real part is used. Since this expression avoids any eigenvalue tracing and only requires the sum of the eigenvalues at each image it is numerically more stable for few images or low energies. It can be expected to be accurate for large frequencies and/or large $T_{0}$, i.e., when the approximation in equation $(29)$ is valid.

\section{The product of eigenvalues of the full Hessian to approximate $\sigma$}

The numerically most stable fall-back option we found is to use the Hessian of the full instanton, i.e., the matrix of second derivatives of the Euclidean action with respect to all atom coordinates of all images. This matrix is required to calculate the temperature-dependent rate constant directly ("canonical instanton")!25 The eigenvalues $\lambda_{i}$ of that full Hessian accounts for the fluctuations perpendicular and along the instanton path. It is 
not directly possible to obtain $u_{i}$ from these, but since $\sigma$ covers the fluctuations perpendicular to the path, this is available by projection.

The eigenvectors associated to the $\lambda_{i}$ are denoted by $v_{i}$. One $\lambda$ is zero, its eigenvector $v_{\text {tang }}$ provides the tangent to the instanton path. It is scaled to have unit-length for each image. $\left\langle v_{i} \mid v_{\text {tang }}\right\rangle$ is the projection of an arbitrary eigenvector on the tangent. It is between between 0 and 1 for each eigenvector, $\sum_{i=1}^{N P-1}\left\langle v_{i} \mid v_{\text {tang }}\right\rangle^{2}=P-1$. With that, the fluctuations perpendicular to the path result in

$$
\exp (\sigma)=\left(\frac{\beta}{P}\right)^{(N-1) P} \prod_{i=1}^{N P-1} \sqrt{\left|\lambda_{\text {inst }, i}\right|^{1-\left\langle v_{i} \mid v_{\text {tang }}\right\rangle^{2}}}
$$

where $N$ is the number of degrees of freedom in the system, not the number of atoms. Equation (34) is correct if there are no zero modes due to rotation and translation. If there are, however, they have to be taken out of the product, this could be done via similar projections as in equation (34). However, we use the analytical expression of the eigenvalues of the instanton-Hessian relating to zero vibrational frequencies:

$$
\lambda_{0, i}=4\left(\frac{P}{\beta}\right)^{2} \sin ^{2}(i \pi / P) \quad i=1, \ldots, P
$$

The product of all these eigenvalues except the last one which is zero is termed $A_{0}$ :

$$
A_{0}=\prod_{i=1}^{P-1} \lambda_{0, i}
$$

with that, for $N_{0}$ zero modes $\left(N_{0}=N-D\right)$

$$
\begin{aligned}
\exp (\sigma)=\left(\frac{\beta}{P}\right)^{\left(N-1-N_{0}\right) P} \frac{1}{A_{0}^{N_{0}}} \times & \prod_{i=1}^{N P-1-N_{0}} \sqrt{\left|\lambda_{\text {inst }, i}\right|^{1-\left\langle v_{i} \mid v_{\text {tang }}\right\rangle^{2}}}
\end{aligned}
$$

where all zero eigenvalues are ignored in the product. From that, we obtain $\sigma^{\prime}$ as $\sigma^{\prime}=\sigma / T_{0}$. This is only accurate if equation $(29)$ is fulfilled, i.e. for large frequencies.

Apart from the methods described here, which turned out to be the most promising ones, we tested several other approaches. For example rather than averaging frequencies, it is possible to average the Hessian matrices $\mathbf{V}^{\prime \prime}$ with the component tangentially to the path removed. The eigenvalues of the resulting averaged Hessian can be used as approximations for $u_{i}(E) / T_{0}(E)$. For an instanton path which couples strongly to other vibrational modes, the removal is not exact, though.

\section{APPLICATIONS}

As a numerical test of our derivations we apply them to two chemical systems, one described by a fitted potential energy surface, the other one with energies, gradients and Hessians calculated on the fly from DFT. As a first test system we chose the reaction $\mathrm{H}_{2}+\mathrm{OH} \rightarrow \mathrm{H}_{2} \mathrm{O}$ $+\mathrm{H}$, which has been investigated in great detail in the literature. $\frac{56 / 72175}{86}$ Among the many potential energy surfaces (PES) available, we use the old one by Schatz and Elgersma. ${ }^{73 / 74}$ The reason is that for this surface, we have "exact" reference data for $P(E)$ from quantum dynamics calculations with a time-independent grid representation and the generalized minimum residual (GMRES) method available. ${ }^{[5 / 76}$ The choice of the potential and the system was made to compare to other methods rather than to provide new physical insight into that particular reaction. The results we obtained are shown in Fig. 4. All calculations were done in DL-FIND 69

The results for $P(E)$ are shown in the left panel of Fig. 4. While it is possible to find instantons for $E<E_{\mathrm{RS}, \mathrm{ZPE}}$, the resulting data are not necessary for the calculation of thermal rate constants (right panel) using equation (24). All methods we tested show a similar dependence of $P$ on $E$. This four-atom system has five stability parameters $u_{i}(E)$ perpendicular to the instanton path. Since here we have an analytical expression for the potential energy surface, we can use many images (up to $P=400$ was used), so that even solving the stability matrix differential equation equation 25 is stable enough. The results are very similar to eigenvalue tracing. In both of these approaches, equation (16) and (17) were used to obtain $P(E)$. Even though $P(E)$ obtained from solving the stability matrix differential equation and eigenvalue tracing look very similar, the resulting thermal rate constants are somewhat different $(\sim 13 \%)$ at low temperature. While this emphasizes that very accurate cumulative reaction probabilities are required to calculate thermal rate constants in the range of deep tunneling, such small differences are probably negligible in practical applications. Obtaining $P(E)$ from equation (20) via $\sigma$ using frequency averaging or obtaining $\sigma$ from the full Hessian of the Euclidean action results in pretty good approximations as well. This is the case even though rather small frequencies perpendicular to the instanton are present, in which case the approximation of equation 29 may be questioned. It seems to work well in practice, though. The smallest frequency at the reactant side of the instanton (in the pre-reactive minimum) is only $173 \mathrm{~cm}^{-1}\left(\omega=7.9 \times 10^{-4}\right.$ a.u. $)$ on the PES we used.

For comparison Fig. 4 and Fig. 6 also show rate constants calculated with harmonic transition state theory (HTST) with the full quantum mechanical partition functions of harmonic oscillators used for all vibrations. Thus, they include ZPE, but no tunneling. Additionally, a curve with HTST corrected for tunneling through a symmetric Eckart barrier (height and $\bar{\omega}_{\text {TS }}$ matched to the PES) is shown.

Our values for the cumulative reaction probability $P(E)$ agree very well with the reference values found by quantum dynamics. Small fluctuations are smoothed out by the thermal averaging. This means that the approximations made in instanton theory, most of all the 

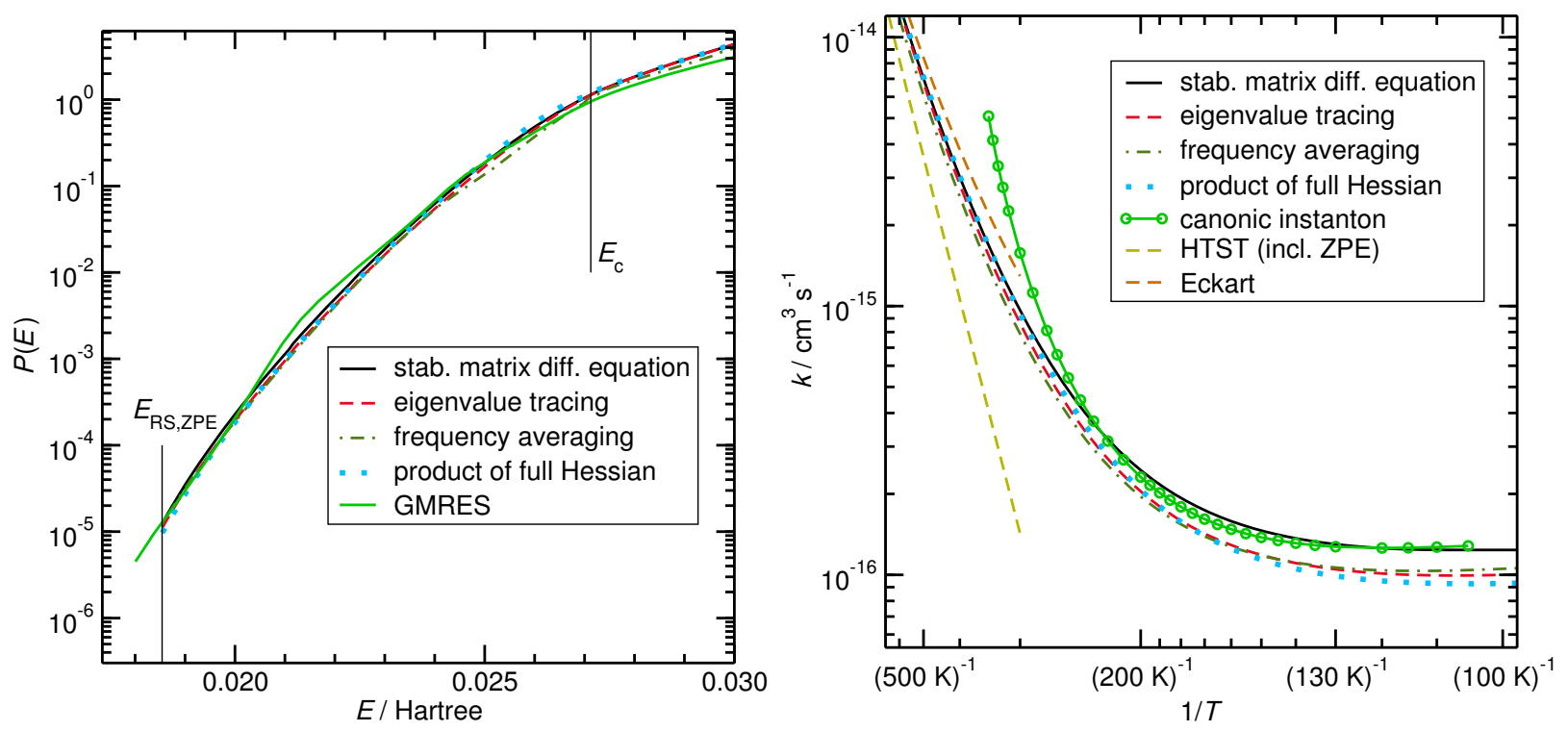

FIG. 4. $P(E)$ for the reaction $\mathrm{H}_{2}+\mathrm{OH}$ on the Schatz-Elgersma PES ${ }^{73 / 74}$ for the different approximations of $\sigma^{\prime}$ or $u_{i}(E)$ discussed in the methods section. GMRES refers to ${ }^{75 / 76}$ and acts as a reference. Thermal rate constants are shown in the left panel.

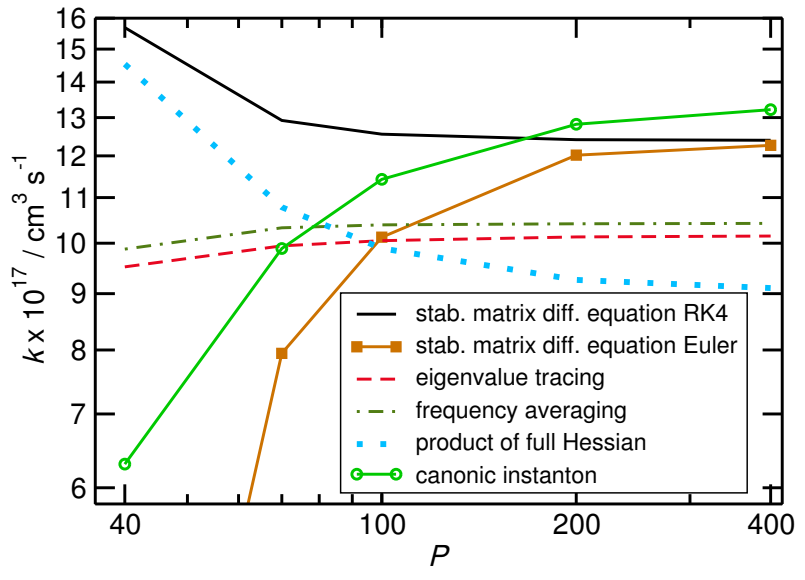

FIG. 5. Robustness of the different methods to calculate the rate constant with change in the number of images $P$ of the instanton. Rate constants at $T=105 \mathrm{~K}$ for the reaction $\mathrm{H}_{2}+\mathrm{OH} \rightarrow \mathrm{H}_{2} \mathrm{O}+\mathrm{H}$ on the Schatz-Elgersma surface ${ }^{73 / 74}$ are compared. The solution of the stability matrix differential equation was performed with a fourth-order Runge-Kutta method (RK4), as well as with the backward Euler approach.

semiclassical approximation, are valid for this system.

A large number of images $P$ can be used for a small system like $\mathrm{H}_{2}+\mathrm{OH} \rightarrow \mathrm{H}_{2} \mathrm{O}+\mathrm{H}$, especially when using a fitted potential energy surface. When the energies, gradients, and Hessians must be calculated on the fly by electronic structure theory, however, $P$ is limited. Therefore, we checked how strongly the thermal rate constant obtained via equation $(24)$ with $P(E)$ calculated with the different methods depends on $P$. The results for one temperature $(T=105 \mathrm{~K})$ are shown in Fig. 5, comparison for the full temperature range is given in the supporting information. The number of images $P$ has been kept constant for the whole range of $E_{b}$. It is obvious from Fig. 5 that frequency averaging and eigenvalue tracing are rather stable at few images while $\sigma$ obtained from the product of the full Hessian causes a significant error for small $P$. The solution of the stability matrix differential equation is also somewhat sensitive to the number of images and breaks down for $P=40$ when using the Euler method. It should be noted that canonical instanton results at the same temperature also depend strongly on $P$, which is well-known. $25 \mid 34$

In order to compare our approaches on a yet more realistic calculation, we applied them to the reaction $\mathrm{H}+$ $\mathrm{HNCO} \rightarrow \mathrm{NH}_{2} \mathrm{CO}$ for which energies, gradients and Hessians were obtained on the fly from density functional calculations. The new calculations were done in DL-FIND 69 via ChemShell, 8788 details of the theoretical treatment are given elsewhere ${ }^{47}$ The energy and its derivatives contain numerical noise due to the incompleteness of the $\mathrm{SCF}$ iterations and other approximations. Only $P=40$ images were used to optimize instantons down to $135 \mathrm{~K}$ and $P=78$ below that. Instantons down to $100 \mathrm{~K}$ were used, which is not quite sufficient to obtain $P(E)$ down to $E_{\mathrm{RS}, \mathrm{ZPE}}$. The effect can be seen in Fig. 6 at low energies, $P(E)$ had to be extrapolated. The change in $P$ leads to a noticeable step in $P(E)$. Stable solution of the stability matrix differential equation could only be achieved for high energies, see Fig. 7. The limit of stability is indicated as a thin vertical line. The last value of $u_{i}(E)$ to the right of the line was used at lower $E$. 

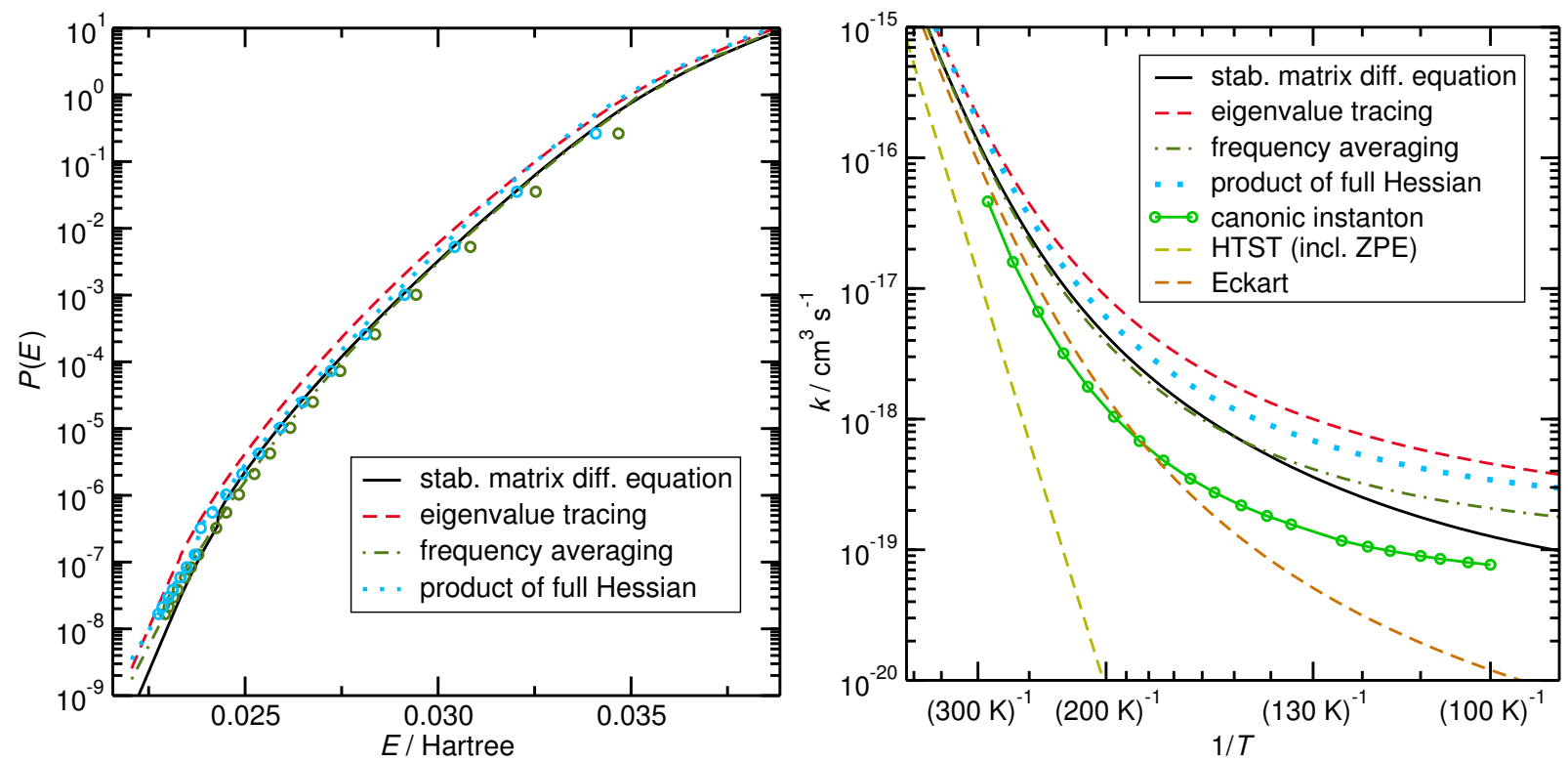

FIG. 6. $P(E)$ and thermal rate constants for the reaction $\mathrm{HNCO}+\mathrm{H} \rightarrow \mathrm{NH}_{2} \mathrm{CO}$ for the different approximations of $\sigma^{\prime}$ or $u_{i}(E)$. In the left graph, open circles show $\left[1+\exp \left(S_{0}\right)\right]^{-1}$ at $E=E_{b}+\sigma / T_{0}$ for each instanton, cf. equation 20 .

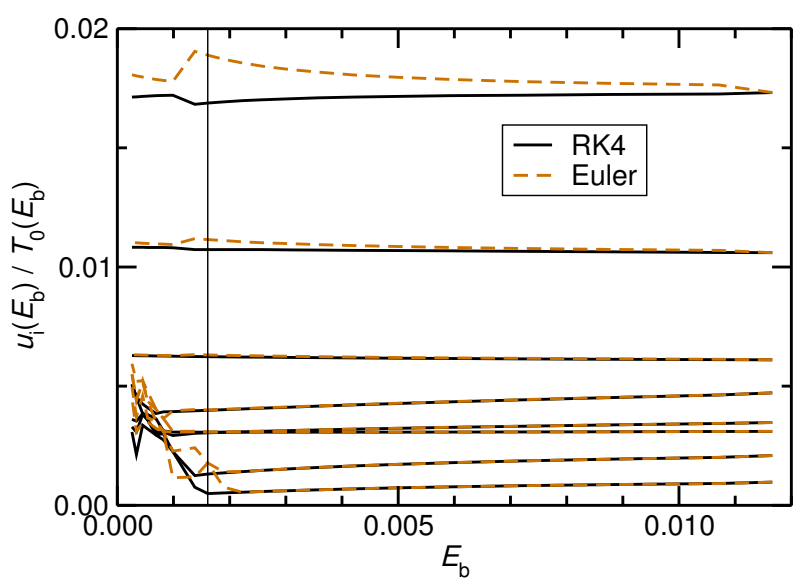

FIG. 7. The stability parameters for the reaction $\mathrm{HNCO}+$ $\mathrm{H} \rightarrow \mathrm{NH}_{2} \mathrm{CO}$ obtained by solving the stability matrix differential equation by a fourth-order Runge-Kutta approach and by the backward Euler algorithm. Both are unstable at lower $E_{b}$ than the thin vertical line.

The canonical rate constants agree reasonably well with those obtained from canonical instanton theory. It is obvious from Fig. 6 that all methods lead to higher rate constants than canonical instanton theory. Overall, it is clear that real-world applications with numerical noise in the potential energy surface and limited $P$ lead to challenges but can be successfully treated with the algorithms presented.

\section{DISCUSSION}

Microcanonical instanton theory allows the calculation of rate constants in bimolecular reactions under lowpressure conditions, i.e. in conditions in which a prereactive minimum will not thermally equilibrate. It also provides thermal rate constants over the full temperature range. The problem of canonical semiclassical instanton theory breaking down at $T_{\mathrm{c}}$ is avoided intrinsically. Both of these advantages offer significant opportunities to study chemical processes at low temperature.

The use of microcanonical instanton theory poses challenges as well, however. In order to calculate any thermal rate constant, instanton calculations along the whole temperature or energy range ( $T_{0}$ corresponds to $\beta \hbar=\hbar /\left(k_{\mathrm{B}} T\right)$ in canonical calculations) from $T_{\mathrm{c}}$ to $E_{b}+\sigma / T_{0}<E_{\mathrm{RS}, \mathrm{ZPE}}$ need to be performed in principle. In practice, one can extrapolate to some extent, as shown for the case of $\mathrm{H}+\mathrm{HNCO} \rightarrow \mathrm{NH}_{2} \mathrm{CO}$ in Fig. 6. Each of these instanton calculations needs to be converged with respect to the number of images $P$. Convergence can be slow, as shown in Fig. 5, but is still generally faster than for canonical instanton theory. In the latter, the rate constant at a specific temperature, as long as this temperature is well below $T_{\mathrm{c}}$, can be provided with high accuracy by converging with respect to $P$. Using microcanonical theory, it is easier to provide a rough approximation of the rate constant over a larger temperature range.

Another important difference between canonical and microcanonical instanton theory is the number of choices of methods an approximations. In canonical instanton theory, over the last years a set of reliable algorithms was established: searching for instantons using a modi- 
fied Newton-Raphson converges fast and reliably, ${ }^{25}$ the rate constant is calculated via the diagonalization of the full Hessian of the Euclidean action $21 \mid 24$ and the rotational partition function, using $J$-shifting, is calculated from the geometries of the images 34 along the instanton path. ${ }^{89}$ The only remaining choice or parameter is the number of images. Rate constants need to be converged with respect to that. In the microcanonical case, such a generally recommendable algorithm is not established yet. Not even a unique, recommendable rate expression is known at present. Equation (15) is the direct consequence of the semiclassical treatment of equation (8), but inapplicable in practice. The combination of equation (16) and equation (17) seems a promising way out, but using equation 20 may be equally justified. Even with a given rate expression, the question of how to calculate the stability parameters $u_{i}(E)$ or their combination $\sigma$ remains. Here, we presented the four most promising approaches of several others we have tried. Practical application to many other cases will have to show which algorithm proves the most promising.

\section{CONCLUSIONS}

Based on different rate expressions for microcanonical instanton rate constants, we have proposed and tested several algorithms to calculate the required stability parameters for non-separable molecular systems, i.e. systems in which the vibrational modes perpendicular to the instanton path couple to vibrations along the path. Any realistic molecules are non-separable. We found the traditional way of integrating the stability matrix differential equation numerically unstable in general. Stability parameters can, however, also be obtained as vibrational frequencies, averaged along the instanton path. With that, the tracing of Hessian eigenvalues along the instanton path and averaging of the corresponding frequencies leads to an accurate and generally more stable algorithm to derive $u_{i}(E)$. An alternative is to average all frequencies and use $\sigma$ rather than the individual $u_{i}(E)$ in the rate expression. In that case, no tracing is necessary, since all Hessian eigenvalues are averaged. Yet another approach is to obtain $\sigma$ from all fluctuations of all images perpendicular to the path by using the full Hessian of the Euclidean action. All methods presented here have their merits in practical applications. Eigenvalue tracing and frequency averaging were shown to be particularly stable at small numbers of images to discretize the instanton path.

Overall, we provide viable approaches to calculate microcanonical instanton rate constants and cumulative reaction probabilities. These have the advantage that they provide rate constants over the whole temperature range without the breakdown of canonical instanton theory at $T_{\mathrm{c}}$ and its inaccuracies close to it.

\section{SUPPLEMENTARY MATERIAL}

Graphs showing the dependence of the rate constants on the number of images for the different approximations.

\section{ACKNOWLEDGMENTS}

We thank Prof. Uwe Manthe for providing the original values of $P(E)$ for the quantum dynamics results reported a long time ago! $! \frac{75 \mid 76}{}$ This work was financially supported by the European Union's Horizon 2020 research and innovation programme (grant agreement No. 646717, TUNNELCHEM). AL received financial support by the Carl-Zeiss foundation.

${ }^{2}$ E. P. Wigner, Z. Phys. Chem. 15, 203 (1932).

${ }^{3}$ H. A. Kramers, Physica 7, 284 (1940)

${ }^{4}$ P. Hänggi, P. Talkner, and M. Borkovec, Rev. Mod. Phys. 62, 251 (1990)

${ }^{5}$ E. Pollak and P. Talkner, Chaos 15, 026116 (2005)

${ }^{6}$ J. Meisner and J. Kästner, Angew. Chem. Int. Ed. 55, 5400 (2016)

'J. S. Langer, Ann. Phys. (N.Y.) 41, 108 (1967)

${ }^{8}$ J. S. Langer, Ann. Phys. (N.Y.) 54, 258 (1969)

${ }^{9}$ W. H. Miller, J. Chem. Phys. 62, 1899 (1975)

${ }^{10}$ S. Coleman, Phys. Rev. D 15, 2929 (1977).

${ }^{11}$ C. G. Callan Jr. and S. Coleman, Phys. Rev. D 16, 1762 (1977)

${ }^{12}$ E. Gildener and A. Patrascioiu, Phys. Rev. D 16, 423 (1977)

${ }^{13}$ I. Affleck, Phys. Rev. Lett. 46, 388 (1981)

${ }^{14}$ S. Coleman, Nucl. Phys. B 298, 178 (1988)

${ }^{15}$ V. A. Benderskii, D. E. Makarov, and C. A. Wight, Adv. Chem. Phys. 88, 55 (1994)

${ }^{10}$ M. Messina, G. K. Schenter, and B. C. Garrett, J. Chem. Phys. 103, 3430 (1995)

${ }^{17}$ S. C. Althorpe, J. Chem. Phys. 134, 114104 (2011)

${ }^{18}$ J. O. Richardson and S. C. Althorpe, J. Chem. Phys. 131, 214106 (2009)

${ }^{19}$ J. O. Richardson and S. C. Althorpe, J. Chem. Phys. 134, 054109 (2011)

${ }^{20}$ J. O. Richardson, J. Chem. Phys. 144, 114106 (2016)

${ }^{21}$ A. Arnaldsson, Calculation of quantum mechanical rate constants directly from ab initio atomic forces, Ph.D. thesis, University of Washington (2007).

${ }^{22}$ H. Jónsson, Proc. Nat. Acad. Sci. U.S.A. 108, 944 (2010)

${ }^{23}$ D. M. Einarsdóttir, A. Arnaldsson, F. Óskarsson, and H. Jónsson, Lect. Notes Comput. Sci. 7134, 45 (2012)

${ }^{24}$ J. B. Rommel, T. P. M. Goumans, and J. Kästner, J. Chem. Theory Comput. 7, 690 (2011)

${ }^{20}$ J. B. Rommel and J. Kästner, J. Chem. Phys. 134, 184107 (2011)

${ }^{20}$ J. B. Rommel, Y. Liu, H.-J. Werner, and J. Kästner, J. Phys. Chem. B 116, $13682(2012)$

${ }^{27}$ S. Chapman, B. C. Garrett, and W. H. Miller, J. Chem. Phys. 63, $2710(1975)$

${ }^{28}$ G. Mills and H. Jónsson, Phys. Rev. Lett. 72, 1124 (1994)

${ }^{29}$ G. Mills, H. Jónsson, and G. K. Schenter, Surf. Sci. 324, 305 (1995)

${ }^{30}$ G. Mills, G. K. Schenter, D. E. Makarov, and H. Jónsson, Chem. Phys. Lett. 278, 91 (1997)

${ }^{31}$ W. Siebrand, Z. Smedarchina, M. Z. Zgierski, and A. FernándezRamos, Int. Rev. Phys. Chem. 18, 5 (1999)

32 Z. Smedarchina, W. Siebrand, A. Fernández-Ramos, and Q. Cui, J. Am. Chem. Soc. 125, 243 (2003)

${ }^{33}$ T. Qian, W. Ren, J. Shi, W. E, and P. Sheng, Physica A 379, 491 (2007) 
${ }^{34}$ S. Andersson, G. Nyman, A. Arnaldsson, U. Manthe, and H. Jónsson, J. Phys. Chem. A 113, 4468 (2009)

${ }^{35}$ T. P. M. Goumans and S. Andersson, Mon. Not. R. Astron. Soc. 406, $2213(2010)$

${ }^{36}$ T. P. M. Goumans, Mon. Not. R. Astron. Soc. 415, 3129 (2011)

${ }^{37}$ T. P. M. Goumans, Mon. Not. R. Astron. Soc. 413, 2615 (2011)

${ }^{38}$ T. P. M. Goumans and J. Kästner, Angew. Chem. Int. Ed. 49, $7350(2010)$

${ }^{3 y}$ J. Meisner, J. B. Rommel, and J. Kästner, J. Comput. Chem. 32, 3456 (2011)

${ }^{40}$ T. P. M. Goumans and J. Kästner, J. Phys. Chem. A 115, 10767 (2011)

${ }^{41}$ M. Kryvohuz and R. A. Marcus, J. Chem. Phys 137, 134107 (2012)

${ }^{42}$ J. Kästner, Chem. Eur. J. 19, 8207 (2013)

${ }^{43}$ S. Álvarez-Barcia, J. R. Flores, and J. Kästner, J. Phys. Chem. A 118, 78 (2014)

${ }^{44}$ M. Kryvohuz, J. Phys. Chem. A 118, 535 (2014)

${ }^{45}$ J. Meisner and J. Kästner, J. Chem. Phys. 144, 174303 (2016).

${ }^{46}$ S. Álvarez-Barcia, M.-S. Russ, J. Meisner, and J. Kästner, Faraday Disc. 195, 69 (2016)

${ }^{47}$ L. Song and J. Kästner, Phys. Chem. Chem. Phys. 18, 29278 (2016)

${ }^{48}$ T. Lamberts, P. K. Samanta, A. Köhn, and J. Kästner, Phys. Chem. Chem. Phys. 18, 33021 (2016)

${ }^{49}$ T. Lamberts, G. Fedoseev, J. Kästner, S. Ioppolo, and H. Linnartz, Astron. Astrophys. , DOI 10.1051/0004 (2017).

${ }^{50}$ P. Hänggi and W. Hontscha, J. Chem. Phys. 88, 4094 (1988)

${ }^{51}$ M. Kryvohuz, J. Chem. Phys. 134, 114103 (2011)

${ }^{52}$ M. Kryvohuz, J. Chem. Phys. 138, 244114 (2013)

${ }^{53}$ Y. Zhang, J. B. Rommel, M. T. Cvitaš, and S. C. Althorpe, Phys. Chem. Chem. Phys. 16, 24292 (2014)

${ }^{54}$ J. O. Richardson, Faraday Disc. 195, 49 (2016)

${ }^{55}$ T. Seideman and W. H. Miller, J. Chem. Phys. 96, 4412 (1992)

${ }^{56}$ W. H. Miller, J. Chem. Phys. A 102, 793 (1998)

${ }^{57}$ W. H. Miller, S. D. Schwartz, and J. W. Tromp, J. Chem. Phys. 79, 4889 (1983)

${ }^{58}$ V. A. Mandelshtam and H. S. Taylor, J. Chem. Phys. 99, 222 (1993)

${ }^{59}$ D. Thirumalai, B. C. Garrett, and B. J. Berne, J. Chem. Phys. 83, 2972 (1985)

${ }^{{ }^{0}}$ M. C. Gutzwiller, J. Math. Phys. 12, 343 (1971)

${ }^{61}$ R. P. Bell, The tunnel effect in chemistry, 1st ed. (Chapman and Hall (London), 1980).

${ }^{62}$ V. Benderskii and D. Makarov, Phys. Lett. A 161, 535 (1992).

${ }^{63}$ V. Benderskii, D. Makarov, D. Pastur, and P. Grinevich, Chem. Phys. 161, 51 (1992)

${ }^{64}$ K. Takayanagi, Prog. Theor. Phy. 8, 497 (1952).

${ }^{65}$ J. M. Bowman, J. Phys. Chem. 95, 4960 (1991)
${ }^{66}$ S. Garashchuk and J. C. Light, J. Chem. Phys. 113, 9390 (2000)

${ }^{67}$ M. Brack and R. K. Bhaduri, Semiclassical Physics (Westview Press, 2003).

${ }^{68}$ H. Cartarius, J. Main, and G. Wunner, Ann. Phys. 322, 315 (2007).

${ }^{69}$ J. Kästner, J. M. Carr, T. W. Keal, W. Thiel, A. Wander, and P. Sherwood, J. Phys. Chem. A 113, 11856 (2009)

${ }^{70} \mathrm{H}$. Kleinert, Path Integrals in Quantum Mechanics, Statistics, Polymer Physics, and Financial Markets, 5th ed. (World Scientific, 2009).

${ }^{71}$ E. B. Wilson Jr., J. Decius, and P. C. Cross, Molecular Vibrations: The Theory of Infrared and Raman Vibrational Spectra (Dover Publications, 1980).

${ }^{72}$ J. Chen, X. Xu, X. Xu, and D. H. Zhang, J. Chem. Phys. 138, $154301(2013)$

${ }^{75}$ S. P. Walch and T. H. Dunning Jr., J. Chem. Phys. 72, 1303 (1980)

${ }^{74}$ G. C. Schatz and H. Elgersma, Chem. Phys. Lett. 73, 21 (1980).

${ }^{75}$ U. Manthe, T. Seideman, and W. H. Miller, J. Chem. Phys. 99, 10078 (1993)

${ }^{76}$ U. Manthe, T. Seideman, and W. H. Miller, J. Chem. Phys. 101, $4759(1994)$

"D. H. Zhang and J. Z. H. Zhang, J. Chem. Phys. 100, 2697 (1994)

${ }^{78}$ U. Manthe and F. Matzkies, J. Chem. Phys. 113, 5725 (2000)

${ }^{79}$ M. Yang, D. H. Zhang, M. A. Collins, and S.-Y. Lee, J. Chem. Phys. 114, 4759 (2001)

${ }^{\gamma}$ J. M. Chan, J. A. Bollinger, C. L. Grewell, and D. M. Dooley, J. Am. Chem. Soc. 126, 3030 (2004)

${ }^{81}$ S. Bhattacharya, A. N. Panda, and H.-D. Meyer, J. Chem. Phys. 132, $214304(2010)$

${ }^{82}$ B. Fu, E. Kamarchik, and J. M. Bowman, J. Chem. Phys. 133, $164306(2010)$

${ }^{83}$ S. Bhattacharya, A. N. Panda, and H.-D. Meyer, J. Chem. Phys. 135, $194302(2011)$

${ }^{84}$ J. Espinosa-Garcia, L. Bonnet, and J. C. Corchado, Phys. Chem. Chem. Phys. 12, 3873 (2010)

${ }^{8}$ T. L. Nguyen, J. F. Stanton, and J. R. Barker, J. Phys. Chem. A 115, 5118 (2011)

${ }^{86}$ B. Fu and D. H. Zhang, J. Chem. Phys. 142, 064314 (2015)

${ }^{87}$ P. Sherwood, A. H. de Vries, M. F. Guest, G. Schreckenbach, C. R. A. Catlow, S. A. French, A. A. Sokol, S. T. Bromley, W. Thiel, A. J. Turner, S. Billeter, F. Terstegen, S. Thiel, J. Kendrick, S. C. Rogers, J. Casci, M. Watson, F. King, E. Karlsen, M. Sjøvoll, A. Fahmi, A. Schäfer, and C. Lennartz, J. Mol. Struct. (THEOCHEM) 632, 1 (2003)

${ }^{88}$ S. Metz, J. Kästner, A. A. Sokol, T. W. Keal, and P. Sherwood, WIREs Comput. Mol. Sci. 4, 101 (2014)

${ }^{89}$ All these are based on earlier work, but described nicely in the references mentioned here. 